\title{
On Adequate Transversals
}

\author{
Jehan Al-Bar \& James Renshaw \\ School of Mathematics \\ University of Southampton \\ Southampton, SO17 1BJ \\ England \\ Email: j.h.renshaw@maths.soton.ac.uk
}

November 28, 2007

\begin{abstract}
We consider adequate transversals of abundant semigroups and prove that, in a particular case, there is a natural embedding of an inverse transversal within a certain regular subsemigroup. We also introduce the concepts of simplistic, perfect and quasi-adequate transversal and provide a number of interesting connections between these.
\end{abstract}

\section{Introduction and Preliminaries}

The concept of an inverse transversal was introduced in 1978 by Blyth and McAlister and arises in response to natural questions concerning the greatest idempotent in a naturally ordered regular semigroup. At about the same time, Fountain introduced us to abundant and adequate semigroups as very natural generalisations of regular and inverse semigroups. Later El-Qallali, and even later Chen, Luo and others, generalised the notion of transversal to abundant semigroups and it is this generalisation that we consider in this paper.

Let $S^{0}$ be an inverse subsemigroup of a regular semigroup $S$ and suppose that for all $x \in$ $S,\left|S^{0} \cap V(x)\right|=1$. Then $S^{0}$ is called an inverse transversal of $S$ and we denote the unique inverse of $x$ in $S^{0}$ by $x^{0}$. It can be shown that for every $x \in S$ there is a unique (in a sense to be made precise later) factorisation in the form $x=e_{x} x^{00} f_{x}$, where $e_{x}, f_{x} \in E(S)$ and $x^{00}=\left(x^{0}\right)^{0} \in S^{0}$. It is this factorisation that provides the inspiration for the generalisation to adequate transversals below. We shall assume that the reader is familiar with the basic ideas of inverse transversals and a very useful summary account is given by Blyth in [1].

After introductory definitions and results, we consider in section 2, the role of regular elements within an adequate transversal and in particular show that each regular element has a unique inverse within the transversal. If the set of regular elements forms a subsemigroup then the adequate transversal contains a natural copy of an inverse transversal of this regular subsemigroup. In section 3 we introduce two important subsets of $S$, designated $R$ and $L$, and show that they behave in a very similar way to that of the inverse transversal case. We also consider a number of special cases of products of element within adequate transversals and prove a number of results which will be useful in later sections as well as considering the concept of left adequate semigroups. In section 4 we define the notion of a left (right) simplistic transversal and demonstrate that the situation is different from the inverse case in general. Perfect transversals are presented in section 5 and connections between them, multiplicative transversals and quasi-ideals are established. We 
consider quasi-adequate transversals in section 6 by making use of the results on regular elements from section 2. The final section demonstrates that the situation for monoids is very much simpler than the general case, in line with the corresponding situation for inverse transversals.

Unless otherwise stated the terminology and notation will be that of [8]. Let $S$ be a semigroup. Define a left congruence $\mathcal{R}^{*}$ on $S$ by

$$
\mathcal{R}^{*}=\left\{(a, b) \in S \times S \mid x a=y a \text { if and only if } x b=y b \text { for all } x, y \in S^{1}\right\} .
$$

The right congruence $\mathcal{L}^{*}$ is defined dually. It is easy to show that if $a$ and $b$ are regular elements then $a \mathcal{R}^{*} b$ if and only if $a \mathcal{R} b$. We say that a semigroup is abundant if each $\mathcal{R}^{*}$-class and each $\mathcal{L}^{*}$-class contains an idempotent. An abundant semigroup in which the idempotents commute is called adequate. It is then clear that regular semigroups are abundant and that inverse semigroups are adequate. For examples of non-regular abundant and adequate semigroups, and more information about the basic structure of these see [5] and $[6]$.

Lemma 1.1 ([2, Lemma 1.1]) Let $e \in E(S)$ and $a \in S$. Then $e \mathcal{R}^{*} a$ if and only if ea $=a$ and for all $x, y \in S^{1}, x a=y a$ implies $x e=y e$.

Lemma 1.2 ([2, Lemma 1.2]) A semigroup $S$ is adequate if and only if each $\mathcal{L}^{*}-$ class and each $\mathcal{R}^{*}$-class contain a unique idempotent and the subsemigroup generated by $E(S)$ is regular.

If $S$ is an adequate semigroup and $a \in S$ then we shall denote by $a^{*}$ the unique idempotent in $L_{a}^{*}$ and by $a^{+}$the unique idempotent in $R_{a}^{*}$. It is easy to show that if $S$ is adequate and $a, b \in S$ then $a \mathcal{R}^{*} b$ if and only if $a^{+}=b^{+}$and $a \mathcal{L}^{*} b$ if and only if $a^{*}=b^{*}$.

Lemma 1.3 ([2, Lemma 1.3]) If $S$ is an adequate semigroup then for all $a, b \in S,(a b)^{*}=$ $\left(a^{*} b\right)^{*}$ and $(a b)^{+}=\left(a b^{+}\right)^{+}$.

If $S$ is an abundant semigroup and $U$ is an abundant subsemigroup of $S$ then we say that $U$ is a *-subsemigroup of $S$ if $\mathcal{L}^{*}(U)=\mathcal{L}^{*}(S) \cap(U \times U), \mathcal{R}^{*}(U)=\mathcal{R}^{*}(S) \cap(U \times U)$. It can be shown that $U$ is a $*$-subsemigroup of $S$ if and only if for all $a \in U$ there exist $e, f \in E(U)$ such that $e \in L_{a}^{*}(S), f \in R_{a}^{*}(S)$ (see [4]).

Let $S$ be an abundant semigroup and $S^{0}$ be an adequate *-subsemigroup of $S$. We say that $S^{0}$ is an adequate transversal of $S$ if for each $x \in S$ there is a unique $\bar{x} \in S^{0}$ and $e, f \in E$ such that

$$
x=e \bar{x} f \text { and such that } e \mathcal{L} \bar{x}^{+} \text {and } f \mathcal{R} \bar{x}^{*} .
$$

It is straightforward to show, [4], that such an $e$ and $f$ are uniquely determined by $x$. Hence we normally denote $e$ by $e_{x}, f$ by $f_{x}$ and the semilattice of idempotents of $S^{0}$ by $E^{0}$. The following are either well-known or are easy to check and are worth mentioning separately:

Lemma 1.4 Let $S$ be an abundant semigroup with an adequate transversal $S^{0}$. Then for all $x \in S$

$$
\begin{aligned}
& \text { 1. } e_{x} \mathcal{R}^{*} x \text { and } f_{x} \mathcal{L}^{*} x, \\
& \text { 2. if } x \in S^{0} \text { then } e_{x}=x^{+} \in E^{0}, \bar{x}=x, f_{x}=x^{*} \in E^{0} \text {, } \\
& \text { 3. if } x \in E^{0} \text { then } e_{x}=\bar{x}=f_{x}=x \text {, }
\end{aligned}
$$


4. $e_{\bar{x}} \mathcal{L} e_{x}$ and hence $e_{\bar{x}} e_{x}=e_{\bar{x}}$ and $e_{x} e_{\bar{x}}=e_{x}$,

5. $f_{\bar{x}} \mathcal{R} f_{x}$ and hence $f_{\bar{x}} f_{x}=f_{x}$ and $f_{x} f_{\bar{x}}=f_{\bar{x}}$.

Remark 1.5 Notice that $\bar{x}=e_{\bar{x}} \bar{x} f_{\bar{x}}=e_{\bar{x}} e_{x} \bar{x} f_{x} f_{\bar{x}}=e_{\bar{x}} x f_{\bar{x}}$. Hence $\bar{x} f_{x}=e_{\bar{x}} x f_{\bar{x}} f_{x}=$ $e_{\bar{x}} x f_{x}=e_{\bar{x}} x$.

Lemma 1.6 [2, Proposition 2.3] Let $S^{0}$ be an adequate transversal of an abundant semigroup $S$ and let $x, y \in S$. Then

1. $x \mathcal{R}^{*} y$ if and only if $e_{x}=e_{y}$,

2. $x \mathcal{L}^{*} y$ if and only if $f_{x}=f_{y}$.

As mentioned in [4], it can be shown that if $S$ is regular then $S^{0}$ is an inverse transversal of $S$. This also follows easily from the results in section 2 below.

In what follows, unless otherwise stated, we shall assume that $S$ is an abundant semigroup and that $S^{0}$ is an adequate transversal of $S$. We define

$$
I=\left\{e_{x}: x \in S\right\} \quad \Lambda=\left\{f_{x}: x \in S\right\} .
$$

These sets play an important role in the case of inverse transversals and provide a similar function for adequate transversals. Unlike the former case however, they are not in general bands.

Lemma 1.7 [2, Lemma 2.1] Let $S$ be as above. Then

$$
\begin{aligned}
& \text { 1. } I \cap \Lambda=E^{0} \text {, } \\
& \text { 2. } I=\left\{e \in E: \exists l \in E^{0} \quad l \mathcal{L} e\right\} \quad \Lambda=\left\{f \in E: \exists h \in E^{0} \quad h \mathcal{R} f\right\} \\
& \text { 3. } I E^{0} \subseteq I \quad E^{0} \Lambda \subseteq \Lambda \text {. }
\end{aligned}
$$

Given that $S^{0}$ is a adequate $*$-subsemigroup of $S$ then the $l$ and $h$ in the previous lemma are uniquely determined by $e$ and $f$ respectively.

It is easy to show that $I=\left\{x \in S: x=e_{x}\right\}$ and $\Lambda=\left\{x \in S: x=f_{x}\right\}$.

A number of simple observations regarding $x \in I, y \in \Lambda$ are worth noting:

1. $e_{x}=x, \bar{x}=f_{x}=e_{\bar{x}}$,

2. $x \mathcal{L} \bar{x}$ and so $x=x \cdot \bar{x}$ and $\bar{x}=\bar{x} \cdot x$,

3. $e_{y}=\bar{y}=f_{\bar{y}}, f_{y}=y$,

4. $y \mathcal{R} \bar{y}$ and so $y=\bar{y} \cdot y$ and $\bar{y}=y \cdot \bar{y}$.

We also have that for all $x \in S$
5. $\overline{e_{x}}=e_{\bar{x}}=\bar{x}^{+}=f_{e_{x}}$,
6. $\overline{f_{x}}=f_{\bar{x}}=\bar{x}^{*}=e_{f_{x}}$.

It then follows that $I, \Lambda \subseteq I \Lambda$. Notice that it follows from Lemma 1.6 together with some of the preceding observations that $\left|R_{x}^{*} \cap I\right|=\left|L_{x}^{*} \cap \Lambda\right|=1$. 


\section{Regularity}

When studying adequate transversals of abundant semigroups, it is natural to ask about the relationship with inverse transversals of regular semigroups and a natural starting point would be to consider the role of regular elements. In particular we would wish to know about the structure of the set of regular elements within $S$ and the role that their inverses play within the transversal.

Lemma 2.1 I $\subseteq$ Reg $(S)$, the set of regular elements of $S$. Moreover $f_{\bar{x}} e_{\bar{y}} \in V\left(e_{y} f_{x}\right) \cap S^{0}$.

Proof. First $e_{y} f_{x}=e_{y} e_{\bar{y}} f_{\bar{x}} f_{x}=e_{y} f_{\bar{x}} e_{\bar{y}} f_{x}=\left(e_{y} f_{x}\right) f_{\bar{x}} e_{\bar{y}}\left(e_{y} f_{x}\right)$.

Also, $\left(f_{\bar{x}} e_{\bar{y}}\right)\left(e_{y} f_{x}\right)\left(f_{\bar{x}} e_{\bar{y}}\right)=f_{\bar{x}} e_{\bar{y}} f_{\bar{x}} e_{\bar{y}}=f_{\bar{x}} e_{\bar{y}}$.

It follows from [2, Corollary 2.4] that $f_{\bar{x}} e_{\bar{y}}$ is the unique inverse of $e_{y} f_{x}$ in $S^{0}$.

Suppose that $x \in \operatorname{Reg}(S)$. Using the fact that $x \mathcal{R} e_{x}$ and $x \mathcal{L} f_{x}$ then from [8, Theorem 2.3.4] there exists a unique $x^{0} \in V(x)$ with $x x^{0}=e_{x}$ and $x^{0} x=f_{x}$. Notice then that $x^{0}=x^{0} x x^{0}=x^{0} e_{x} \bar{x} f_{x} x^{0}=x^{0} x x^{0} \bar{x} x^{0} x x^{0}=x^{0} \bar{x} x^{0}$. We have therefore proved part of

Theorem 2.2 If $x \in \operatorname{Reg}(S)$ then $\bar{x} \in \operatorname{Reg}(S)$. Moreover $x^{0} \in V(\bar{x})$ and $x^{0}=(\bar{x})^{0}$.

Proof. For any $x \in \operatorname{Reg}(S)$ we have $x^{0} \bar{x}=x^{0} e_{x} \bar{x}=x^{0} e_{x} e_{\bar{x}} x f_{\bar{x}}=x^{0} e_{x} x f_{\bar{x}}=x^{0} x f_{\bar{x}}=$ $f_{x} f_{\bar{x}}=f_{\bar{x}}$ and consequently $\bar{x} x^{0} \bar{x}=\bar{x} f_{\bar{x}}=\bar{x}$ from which we can deduce that $\bar{x} \in \operatorname{Reg}(S)$ and that $x^{0} \in V(\bar{x})$. In a similar way, $\bar{x} x^{0}=e_{\bar{x}}$ and from the uniqueness of $(\bar{x})^{0}$ is follows that $x^{0}=(\bar{x})^{0}$.

In what follows we shall write $x^{00}$ for $\left(x^{0}\right)^{0}$. The following result then demonstrates that the inverse $x^{0}$ has precisely the properties that we would expect from a consideration of the corresponding elements in inverse transversals.

Theorem 2.3 If $x \in \operatorname{Reg}(S)$ then $x^{0} \in S^{0}, \bar{x}=x^{00}$ and $x^{0}=x^{000}$.

Proof. Since $\bar{x} \in V\left(x^{0}\right)$ then it is clear that $x^{0} \bar{x} \mathcal{R} x^{0}$. Moreover $x^{0} \bar{x}=f_{\bar{x}} \in E^{0} \subseteq I$ and so $x^{0} \bar{x}=e_{x^{0}}$ since $\left|I \cap R_{x^{0}}^{*}\right|=1$. In a similar way $\bar{x} x^{0}=f_{x^{0}}$ and so $\bar{x}=x^{00}$ by the uniqueness of $x^{00}$. Since $e_{x^{0}}, f_{x^{0}} \in E^{0}$ then $x^{0} \in S^{0}$. From Theorem 2.2 it follows that $x^{0}=x^{000}$.

Notice that if $x \in I \cup \Lambda$ then $x^{0}=\bar{x}=x^{00}$. Also, by [2, Corollary 2.4] we see that

Theorem 2.4 If $x \in \operatorname{Reg}(S)$ then $\left|V(x) \cap S^{0}\right|=1$.

Let $x \in I$ so that $x \in \operatorname{Reg}(S)$ and $x=e_{x}=x x^{0}$. It is easy to see that

$$
x x^{0}=\left(x x^{0}\right)\left(x^{00} x^{0}\right)\left(x^{00} x^{0}\right) \text { and that } e_{x x^{0}}=x x^{0} .
$$

It follows that

$$
I=\left\{x \in \operatorname{Reg}(S): x=x x^{0}\right\}=\left\{x \in \operatorname{Reg}(S): x^{0}=x^{0} x\right\}=\left\{x x^{0}: x \in \operatorname{Reg}(S)\right\} .
$$

In a similar way,

$$
\Lambda=\left\{x \in \operatorname{Reg}(S): x=x^{0} x\right\}=\left\{x \in \operatorname{Reg}(S): x^{0}=x x^{0}\right\}=\left\{x^{0} x: x \in \operatorname{Reg}(S)\right\} .
$$


Let $T=\operatorname{Reg}(S)$, let $U=T \cap S^{0}$ and suppose that $T$ is a subsemigroup of $S$. It is clear that $U$ is then a regular subsemigroup of $T$. It is also relatively straightforward to see that $U=\left\{x^{0}: x \in T\right\}=\left\{x \in T: x=\bar{x}=x^{00}\right\}$. Given that $S^{0}$ is adequate then we can easily deduce that $U$ is inverse and that $U$ is an inverse transveral of the regular semigroup $T$.

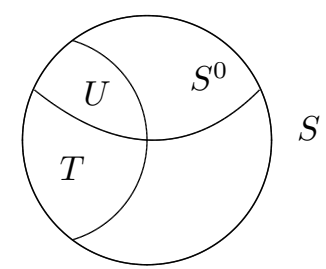

Moreover, it is reasonably clear that, with the obvious notation, $E(T)=E(S), E(U)=$ $E\left(S^{0}\right)$ and $I(T)=I(S), \Lambda(T)=\Lambda(S)$ and so from [1, Theorem 1.3] we see that

Proposition 2.5 If $T$ is a subsemigroup of $S$ then $I$ is a left regular subband of $S$ and $\Lambda$ is a right regular subband of $S$.

Moreover, as in [1, Theorem $1.1 \&$ Theorem 1.4] we can deduce

Proposition 2.6 If $T$ is a subsemigroup of $S$ then for all $x, y \in T$

$$
(x y)^{0}=\left(x^{0} x y\right)^{0} x^{0}=y^{0}\left(x y y^{0}\right)^{0}=y^{0}\left(x^{0} x y y^{0}\right)^{0} x^{0},
$$

and

$$
\left(x y^{0}\right)^{0}=y^{00} x^{0},\left(x^{0} y\right)^{0}=y^{0} x^{00} .
$$

The following is a generalisation of [4, Example 2.2] and provides a mechanism for extending adequate transversals. In particular given an inverse transversal $U$ of a regular semigroup $T$ (so that $U$ is an adequate transversal of the abundant semigroup $T$ ) there is no unique abundant semigroup $S$ with adequate transversal $S^{0}$ such that $U=T \cap S^{0}$.

Suppose that $S^{0}$ is an adequate transversal of an abundant semigroup $S$ and suppose also that $M$ is a cancellative monoid with identity 1 . Then it is straighforward to show that $M \times S^{0}$ is an adequate transversal of the abundant semigroup $M \times S$. In fact $E(M \times S)=$ $\{1\} \times E(S)$ and $E\left(M \times S^{0}\right)=\{1\} \times S^{0}$ and it is easy to show that $(n, b) \in L_{(m, a)}^{*}(M \times S)$ if and only if $b \in L_{a}^{*}(S)$. Moreover $\overline{(m, a)}=(m, \bar{a}), e_{(m, a)}=\left(1, e_{a}\right)$ and $f_{(m, a)}=\left(1, f_{a}\right)$.

An obvious question would be "Given a regular semigroup $T$ and an adequate semigroup $S^{0}$ whose intersection, $U$, is inverse, can we construct an abundant semigroup $S$ such that $S^{0}$ is an adequate transversal of $S$ and such that $T$ is the subsemigroup of regular elements of $S$ ? Moreover, is such an $S$ unique?"

\section{Adequate Transversals}

We define the following two important subsets of $S$.

$$
R=\left\{x \in S: e_{x}=e_{\bar{x}}\right\}, L=\left\{x \in S: f_{x}=f_{\bar{x}}\right\}
$$

These sets can in fact be described in a number of different ways.

Theorem 3.1

$$
\begin{gathered}
R=\left\{x \in S: e_{x} \in E^{0}\right\}=\left\{x \in S: x=\bar{x} f_{x}\right\}=\left\{x \in S: x=e_{\bar{x}} x\right\} \\
=\left\{x \in S: x \mathcal{R}^{*} \bar{x}\right\}=\left\{x \in S: \bar{x}=e_{x} \bar{x}\right\} .
\end{gathered}
$$


Proof. First notice that from Lemma 1.6 and Remark 1.5 if follows that $R=\{x \in S$ : $\left.x \mathcal{R}^{*} \bar{x}\right\}$ and that $\left\{x \in S: x=\bar{x} f_{x}\right\}=\left\{x \in S: x=e_{\bar{x}} x\right\}$.

If $x \in R$ then $x=e_{x} \bar{x} f_{x}=e_{\bar{x}} \bar{x} f_{x}=\bar{x} f_{x}$. If $x=\bar{x} f_{x}$ then $e_{x} \bar{x} f_{x}=e_{\bar{x}} \bar{x} f_{x}$ and so by uniqueness of $e_{x}$ it follows that $e_{x}=e_{\bar{x}} \in E^{0}$. Now if $e_{x} \in E^{0} \subseteq S^{0}$ then $\bar{x}=e_{\bar{x}} \bar{x}=\overline{e_{x}} \bar{x}=$ $e_{x} \bar{x}$. Finally, if $\bar{x}=e_{x} \bar{x}$ then $e_{x} \bar{x} f_{x}=\bar{x} f_{x}=e_{\bar{x}} \bar{x} f_{x}$ and so $e_{x}=e_{\bar{x}}$ by uniqueness of $e_{x}$ and $x \in R$. Hence we have demonstrated that

$$
R \subseteq\left\{x \in S: x=\bar{x} f_{x}\right\} \subseteq\left\{x \in S: e_{x} \in E^{0}\right\} \subseteq\left\{x \in S: \bar{x}=e_{x} \bar{x}\right\} \subseteq R .
$$

Theorem 3.2 $L \cap R=S^{0}$.

Proof. If $x \in L \cap R$ then $f_{x} \in E^{0}$ and $e_{x} \in E^{0}$. Hence $x=e_{x} \bar{x} f_{x} \in S^{0}$.

Conversely, if $x \in S^{0}$ then $e_{x}=x^{+} \in E^{0}$ and $f_{x}=x^{*} \in E^{0}$ and so $x \in L \cap R$.

Theorem 3.3 $I=L \cap E(S)$.

Proof. If $x \in I$ then $f_{x}=\bar{x} \in E^{0}$ and so $x \in L$.

Conversely, if $x \in L \cap E(S)$ then $f_{x} \in E^{0}$. Moreover, $x \mathcal{L}^{*} f_{x}$ and since $x, f_{x} \in E(S)$ it follows that $x \mathcal{L} f_{x}$ and so $x \in I$ from Lemma 1.7 .

It follows that, as with inverse transversals, we have a diagram

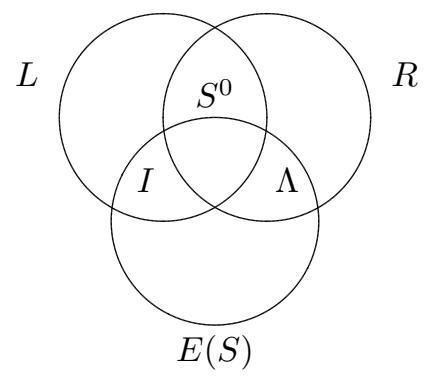

Calculating the values of $e_{x y}, f_{x y}$ and $\overline{x y}$ in terms of $x$ and $y$ for a given $x, y \in S$ seems to be particularly difficult but in some special cases we can make progress.

Lemma 3.4 Let $x \in R, y \in S, z \in L$. Then

$$
\begin{aligned}
& \text { 1. } e_{e_{y} x}=e_{y} e_{x}, \quad f_{z f_{y}}=f_{z} f_{y}, \\
& \text { 2. } \overline{e_{y} x}=e_{\bar{y}} \bar{x}, \overline{z f_{y}}=\bar{z} f_{\bar{y}}, \\
& \text { 3. } f_{e_{y} x}=\left(e_{\bar{y}} \bar{x}\right)^{*} f_{x}, \quad e_{z f_{y}}=e_{z}\left(\bar{z} f_{\bar{y}}\right)^{+} \text {. }
\end{aligned}
$$

Proof. We prove only the equations involving $x$ and $y$, the others being similar. Notice first that $x=e_{x} \bar{x} f_{x}$ and so

$$
e_{y} x=e_{y} e_{x} \bar{x} f_{x}=e_{y} e_{\bar{y}} e_{x} \bar{x} f_{x}=e_{y} e_{x}\left(e_{\bar{y}} \bar{x}\right) f_{x}=e_{y} e_{x}\left(e_{\bar{y}} \bar{x}\right)\left(\left(e_{\bar{y}} \bar{x}\right)^{*} f_{x}\right) .
$$

We use the fact that $\left|I \cap R_{w}^{*}\right|=1$ for all $w \in S$. Now $e_{x} \mathcal{R}^{*} x$ and so $e_{y} e_{x} \mathcal{R}^{*} e_{y} x$. Moreover $e_{y} e_{x} \in I$ since $I E^{0} \subseteq I$ and so $e_{e_{y} x}=e_{y} e_{x}$. 
We now show that $e_{e_{y} x}=e_{y} e_{x} \mathcal{L}\left(e_{\bar{y}} \bar{x}\right)^{+}$. Notice first that $e_{y} \mathcal{L} e_{\bar{y}}$ and so $e_{y} e_{x} \mathcal{L} e_{\bar{y}} e_{x}$. We also have $e_{\bar{x}} \mathcal{R}^{*} \bar{x}$ and so $e_{\bar{y}} e_{\bar{x}} \mathcal{R}^{*} e_{\bar{y}} \bar{x}$ and since $e_{\bar{y}} e_{\bar{x}} \in E^{0}$ then $e_{\bar{y}} e_{\bar{x}}=\left(e_{\bar{y}} \bar{x}\right)^{+}$. But if $x \in R$ then $e_{x}=e_{\bar{x}}$ and so $e_{y} e_{x} \mathcal{L} e_{\bar{y}} e_{x}=e_{\bar{y}} e_{\bar{x}}=\left(e_{\bar{y}} \bar{x}\right)^{+}$as required.

We now show that $\left(e_{\bar{y}} \bar{x}\right)^{*} \mathcal{R}\left(e_{\bar{y}} \bar{x}\right)^{*} f_{x}$. We have $f_{x} \mathcal{R}^{*} \bar{x}^{*}$ and so $\left(e_{\bar{y}} \bar{x}\right)^{*} f_{x} \mathcal{R}^{*}\left(e_{\bar{y}} \bar{x}\right)^{*} \bar{x}^{*}$. But $\left(e_{\bar{y}} \bar{x}\right)^{*} \bar{x}^{*}=\left(e_{\bar{y}} \bar{x}\right)^{*}$ since $\left(e_{\bar{y}} \bar{x}\right)^{*} \mathcal{L}^{*}\left(e_{\bar{y}} \bar{x}\right)$ and hence $\left(e_{\bar{y}} \bar{x}\right)^{*} \bar{x}^{*} \mathcal{L}^{*}\left(e_{\bar{y}} \bar{x}\right) \bar{x}^{*}=\left(e_{\bar{y}} \bar{x}\right)$ and consequently $\left(e_{\bar{y}} \bar{x}\right)^{*} \bar{x}^{*}=\left(e_{\bar{y}} \bar{x}\right)^{*}$ by uniqueness of idempotents in $\mathcal{L}^{*}$-classes.

There are a number of special cases that can easily be deduced from this. For example:

\section{Corollary 3.5}

1. If $x \in S^{0}$ then $e_{y} x^{+}=e_{e_{y} x}$ and $x^{*} f_{y}=f_{x f_{y}}$.

2. If $x \in \Lambda$ then $e_{y} \bar{x}=e_{e_{y} x}$. If $x \in I$ then $\bar{x} f_{y}=f_{x f_{y}}$.

3. If $x, y \in S^{0}$ then $y^{+} x^{+}=e_{y^{+} x}$ and $x^{*} y^{*}=f_{x y^{*}}$.

4. If $x y \in R$ then $e_{x} e_{x y}=e_{x y}$. If $x y \in L$ then $f_{x y} f_{y}=f_{x y}$.

5. If $x \in S$ then $e_{\bar{x} f_{x}}=e_{\bar{x}}$ and $f_{e_{x} \bar{x}}=f_{\bar{x}}$. Hence $\bar{x} f_{x} \in R$ and $e_{x} \bar{x} \in L$ for all $x \in S$.

Notice from part 5 that we can deduce

\section{Corollary 3.6}

1. $R=\left\{\bar{x} f_{x}: x \in S\right\}, L=\left\{e_{x} \bar{x}: x \in S\right\}$,

2. for all $x \in S, x=e_{x}\left(\bar{x} f_{x}\right) \in L R$ and so $L R=S$.

In particular from Lemma 3.4 we see that if $x \in I, y \in \Lambda$ then $e_{x y}=x e_{y}=x \bar{y}, f_{x y}=$ $(\bar{x} \bar{y})^{*} y=\bar{x} \bar{y} y=\bar{x} y, \overline{x y}=\bar{x} \bar{y}$. Consequently, $\overline{x y} \in E^{0}$. Conversely, if $x \in S$ is such that $\bar{x} \in E^{0}$ then $x=\left(e_{x} \bar{x}\right) f_{x} \in I \Lambda$. Hence we have shown that as with inverse transversals

Theorem 3.7 I $\Lambda=\left\{x \in S: \bar{x} \in E^{0}\right\}$.

Corollary $3.8 S^{0} \cap I \Lambda=E^{0}$.

As another special case, let $x, y \in S$ and suppose that in what follows $f_{x} e_{y} \in E(S)$. Then,

$$
\begin{aligned}
f_{x} e_{y} & =f_{x} e_{y} f_{x} e_{y} \\
& =f_{x} e_{y} e_{\bar{y}} f_{\bar{x}} f_{x} e_{y} \\
& =f_{x} e_{y} f_{\bar{x}} e_{\bar{y}} f_{x} e_{y} \\
& =\left(f_{x} e_{y} f_{\bar{x}}\right)\left(f_{\bar{x}} e_{\bar{y}}\right)\left(e_{\bar{y}} f_{x} e_{y}\right) .
\end{aligned}
$$

Notice that $\left(f_{\bar{x}} e_{\bar{y}}\right)^{+}=\left(f_{\bar{x}} e_{\bar{y}}\right)^{*}=e_{\bar{y}} f_{\bar{x}}$.

Lemma $3.9 f_{x} e_{y} f_{\bar{x}} \mathcal{L} e_{\bar{y}} f_{\bar{x}}$ if and only if $e_{y} f_{x} \in E(S)$.

Proof. First notice that $e_{y} \mathcal{L} e_{\bar{y}}$ and $f_{x} \mathcal{R} f_{\bar{x}}$. Now $\left(f_{x} e_{y} f_{\bar{x}}\right)\left(f_{x} e_{y} f_{\bar{x}}\right)=f_{x} e_{y} f_{x} e_{y} f_{\bar{x}}=$ $f_{x} e_{y} f_{\bar{x}}$, since $f_{x} e_{y} \in E(S)$. Hence $f_{x} e_{y} f_{\bar{x}} \in E(S)$ and so $f_{x} e_{y} f_{\bar{x}} \mathcal{L} e_{\bar{y}} f_{\bar{x}}$ if and only if $\left(f_{x} e_{y} f_{\bar{x}}\right)\left(e_{\bar{y}} f_{\bar{x}}\right)=f_{x} e_{y} f_{\bar{x}}$ and $\left(e_{\bar{y}} f_{\bar{x}}\right)\left(f_{x} e_{y} f_{\bar{x}}\right)=e_{\bar{y}} f_{\bar{x}}$. Now the first of these equations is always true and so $f_{x} e_{y} f_{\bar{x}} \mathcal{L} e_{\bar{y}} f_{\bar{x}}$ if and only if $\left(e_{\bar{y}} f_{\bar{x}}\right)\left(f_{x} e_{y} f_{\bar{x}}\right)=e_{\bar{y}} f_{\bar{x}}$. But if $\left(e_{\bar{y}} f_{\bar{x}}\right)\left(f_{x} e_{y} f_{\bar{x}}\right)=e_{\bar{y}} f_{\bar{x}}$ then $e_{y}\left(e_{\bar{y}} f_{\bar{x}}\right)\left(f_{x} e_{y} f_{\bar{x}}\right) f_{x}=e_{y} e_{\bar{y}} f_{\bar{x}} f_{x}$ and so $e_{y} f_{x} e_{y} f_{x}=e_{y} f_{x}$. Conversely if $e_{y} f_{x} e_{y} f_{x}=e_{y} f_{x}$ then $e_{\bar{y}} e_{y} f_{x} e_{y} f_{x} f_{\bar{x}}=e_{\bar{y}} e_{y} f_{x} f_{\bar{x}}$ and so $\left(e_{\bar{y}} f_{\bar{x}}\right)\left(f_{x} e_{y} f_{\bar{x}}\right)=e_{\bar{y}} f_{\bar{x}}$. Hence the result. 
In a similar way we have

Lemma $3.10 e_{\bar{y}} f_{x} e_{y} \mathcal{R} e_{\bar{y}} f_{\bar{x}}$ if and only if $e_{y} f_{x} \in E(S)$.

We have therefore demonstrated the following result which will prove useful in section 6 ,

Theorem 3.11 Suppose that $l \in \Lambda, i \in I$ and suppose also that $l i, i l \in E(S)$. Then
1. $e_{l i}=l i \bar{l}$;
2. $\overline{l i}=\bar{l} \bar{i}$
3. $f_{l i}=\bar{i} l i$.

As a final special case, consider [9, Proposition 2.1], and notice that the proof of that result holds for all transversals providing $\bar{x} f_{x} e_{y} \bar{y}, \bar{x} f_{x} e_{y}, f_{x} e_{y} \bar{y} \in S^{0}$. Hence

Theorem 3.12 (Cf. [9, Proposition 2.1]) Let $S$ be an abundant semigroup with an adequate transversal $S^{0}$ and let $x, y \in S$. Suppose that $\bar{x} f_{x} e_{y}, f_{x} e_{y} \bar{y} \in S^{0}$. Then
1. $\overline{x y}=\bar{x} f_{x} e_{y} \bar{y}$,
2. $e_{x y}=e_{x}\left(\bar{x} f_{x} e_{y}\right)^{+}$,
3. $f_{x y}=\left(f_{x} e_{y} \bar{y}\right)^{*} f_{y}$.

Notice that in particular this result is true if $x \in L$ and $y \in R$ :

Corollary 3.13 For all $x \in L, y \in R$

$$
\overline{x y}=\bar{x} \bar{y}, \quad e_{x y}=e_{x}(\bar{x} \bar{y})^{+}, \quad f_{x y}=(\bar{x} \bar{y})^{*} f_{y} .
$$

Proof. Let $x \in L, y \in R$ so that $\overline{x y}=\bar{x} f_{x} e_{y} \bar{y}=\bar{x} \bar{y}$. Also, $e_{x y}=e_{x}\left(\bar{x} f_{x} e_{y}\right)^{+}=$ $e_{x}\left(\bar{x} e_{\bar{y}}\right)^{+}=e_{x}\left(\bar{x} \bar{y}^{+}\right)^{+}=e_{x}(\bar{x} \bar{y})^{+}$. Finally, $f_{x y}=\left(f_{x} e_{y} \bar{y}\right)^{*} f_{y}=\left(\bar{x}^{*} \bar{y}\right)^{*} f_{y}=(\bar{x} \bar{y})^{*} f_{y}$.

Say that $S$ is left adequate if $S$ is abundant and every $\mathcal{R}^{*}(S)$-class contains a unique idempotent.

Theorem 3.14 The following are equivalent:
2. $\Lambda=E^{0}$;
3. $R=S^{0}$;
4. $S=L$;
5. $E=I$.

1. $S$ is left adequate,

Proof. $\quad(1) \Rightarrow(2)$. Suppose that $S$ is left adequate and let $x \in \Lambda$. Then $e_{x}=\bar{x} \in E^{0}$. Moreover $f_{x} \mathcal{R} \bar{x}^{*}$ and so $f_{x} \mathcal{R}^{*} \bar{x}^{*}$ and so $f_{x}=\bar{x}^{*} \in E^{0}$. Hence $x=e_{x} \bar{x} f_{x} \in E^{0}$ as required. $(2) \Rightarrow(3)$. If $x \in R$ then $x=\bar{x} f_{x} \in S^{0} E^{0} \subseteq S^{0}$.

$(3) \Rightarrow(4)$. If $x \in S$ then $f_{x} \in \Lambda \subseteq R$ and so $f_{x} \in S^{0} \cap E(S)=E^{0}$. Hence $x \in L$.

$(4) \Rightarrow(5)$. If $x \in E$ then $x \in E \cap L=I$.

$(5) \Rightarrow(1)$. If $e, f \in E(S)$ are such that $e \mathcal{R}^{*} f$ then $e, f \in I$ and so $e=f$ since each $\mathcal{R}^{*}$-class contains exactly one element of $I$. 
If $S$ is adequate then it is clearly both right and left adequate and so $I=\Lambda=E^{0}$. Hence for any $x \in S, x=e_{x} \bar{x} f_{x} \in E^{0} S^{0} E^{0} \subseteq S^{0}$ and so $S=S^{0}$. The converse is clearly true as well. If $S=S^{0}$ then for any $x \in S, e_{x}=x^{+} \in E^{0}, f_{x}=x^{*} \in E^{0}$ and so $S$ is adequate. Hence we have shown

Proposition 3.15 $S$ is adequate if and only if $S=S^{0}$.

\section{Simplistic Transversals}

We say that $S^{0}$ is multiplicative if $\Lambda I \subseteq E^{0}$ and that $S^{0}$ is a quasi-ideal of $S$ if $S^{0} S S^{0} \subseteq S^{0}$ or equivalently [2, Proposition 2.2] if $\Lambda I \subseteq S^{0}$.

Some of the following can also be found in [9, Theorem 2.4].

Lemma 4.1 If $E^{0} I \subseteq E^{0}$ then $I$ is a band.

Proof. If $i, j \in I$ then $i j=i(\bar{i} j) \in I E^{0} \subseteq I$.

Theorem 4.2 The following statements are equivalent:

1. The set I is normal (i.e. for all $i, j, k, l \in I$, ijkl $=i k j l$ );

2. $E^{0}$ is a right ideal of $I$;

3. For all $e \in E^{0}, i \in I$, ei $=e \bar{i}$;

4. I is a left normal band;

5. I is a normal band.

Proof. $\quad(1) \Rightarrow(2)$ Let $i \in I$ and $e \in E^{0}$. Then $e i=e i \bar{i} \bar{i}=e \bar{i} i \bar{i}=e \bar{i} \bar{i}=e \bar{i} \in E^{0}$ and so $E^{0} I \subseteq E^{0}$. Finally $I$ is a band from Lemma 4.1 .

$(2) \Rightarrow(3)$ Let $e \in E^{0}, i \in I$. Then $i e \in I \subseteq E$ (by Lemma 1.7) and hence eie $\in E^{0}$. Since $i \mathcal{L} \bar{i}$ then ie $\mathcal{L} \bar{i}$ e and so eie $\mathcal{L} \bar{i}$. Since $S^{0}$ is adequate then eie $=\bar{i}$. . Hence $e i=e i e i=\bar{i} e i=e \bar{i} i=e \bar{i}$ since $E^{0}$ is a semilattice.

$(3) \Rightarrow(4)$ First, if $e, f \in I$ then $e f=e \bar{e} f=e \bar{e} \bar{f}=e \bar{f} \in I$ (Lemma 1.7) and so $I$ is a band. Let $i, j, k \in I$. Then $i j k=i \bar{i} j k=i \bar{i} \bar{j} \bar{k}=i \bar{i} \bar{k} \bar{j}=i k j$.

$(4) \Rightarrow(5) \Rightarrow(1)$ Obvious.

We shall say that $S^{0}$ is left simplistic if $S^{0} I S^{0} \subseteq S^{0}$. Notice that $S^{0}$ is left simplistic if and only if $S^{0} I \subseteq S^{0}$. In the case of inverse transversals, there are a number of equivalent definitions of this concept (see for example [1]). These however, as we shall see below, are often different in the adequate case.

Let $S^{0}$ be left simplistic and let $x, y \in L$. Notice that $f_{x}, f_{y} \in E^{0}$ and so $f_{x} e_{y} \in S^{0}$. Hence by Theorem 3.12 it follows that $f_{x y}=\left(f_{x} e_{y} \bar{y}\right)^{*} f_{y} \in E^{0}$ and so $x y \in L$. We have therefore demonstrated that

Lemma 4.3 If $S^{0}$ is left simplistic then $L$ is a subsemigroup of $S$.

We suspect that the converse of the previous Lemma is false. However we can now show

Theorem 4.4 The following statements are equivalent: 
1. $S^{0}$ is left simplistic;

2. $S^{0}$ is a right ideal of $L$;

3. $E^{0} I \subseteq S^{0}$.

Proof. $\quad(1) \Rightarrow(2)$ Let $x \in S^{0}, y \in L$. Then $x y=x e_{y} \bar{y} f_{y} \in S^{0} I S_{0} E^{0} \subseteq S^{0} E^{0} \subseteq S^{0}$ as required.

(2) $\Rightarrow(3)$ Let $i \in I$ and $e \in E^{0}$ then $e i=e i \bar{i} \in E^{0} I E^{0} \subseteq S^{0} L S^{0} \subseteq S^{0}$.

$(3) \Rightarrow(1)$ Obvious.

In general the conditions in Theorems 4.2 and 4.4 are distinct. However if $I$ is a band then they do coincide.

Proposition 4.5 If $E^{0} I \subseteq E^{0}$ then $S^{0}$ is left simplistic.

Proof. Let $x, y \in S^{0}$ and $i \in I$ then $x i y=x^{+} x\left(x^{*} i\right) y \in E^{0} S^{0} E^{0} S^{0} \subseteq S^{0}$ as required.

The converse of the previous result is false by [2, Example 2.7].

Theorem 4.6 If I is a band then $S^{0}$ is left simplistic if and only if $E^{0}$ is a right ideal of I;

Proof. It follows that $E^{0} I \subseteq I \subseteq E$ since $I$ is a band. If $e \in E^{0}, i \in I$ then $e i=e i \bar{i} \in$ $S^{0} I S^{0} \subseteq S^{0}$ and so $E^{0} I \subseteq E^{0}$.

Theorem 4.7 $S^{0}$ is both left and right simplistic if and only if $S^{0}$ is a quasi-ideal of $S$.

Proof. Suppose $S^{0}$ is both left and right simplistic and let $x, y \in S^{0}, s \in S$. Then $x s y=x e_{s} \bar{s} f_{s} y \in S^{0} I S^{0} \Lambda S^{0} \subseteq S^{0} \Lambda S^{0} \subseteq S^{0}$.

Conversely, if $S^{0}$ is a quasi-ideal and $x, y \in S^{0}, i \in I$. Then xiy $\in S^{0} I S^{0} \subseteq S^{0} S S^{0} \subseteq S^{0}$ and so $S^{0}$ is left simplistic. Similarly, $S^{0}$ is right simplistic.

We can now conclude

Corollary 4.8 If $S^{0}$ is a quasi-ideal of $S$ then $L$ and $R$ are both subsemigroups of $S$.

\section{$5 \quad$ Perfect Transversals}

Define $S^{0}$ to be a weakly multiplicative adequate transversal of $S$ if $S^{0}$ is an adequate transversal of $S$ and $\overline{\Lambda I} \subseteq E^{0}$, where $\overline{\Lambda I}=\{\overline{l i}: l \in \Lambda, i \in I\}$. We say that $S^{0}$ is perfect if for all $l \in \Lambda, i \in I, e_{l i}=f_{l i}$ and we say that $S^{0}$ is weakly perfect if for all $l \in \Lambda, i \in I, e_{\overline{l i}}=f_{\overline{l i}}$.

Proposition 5.1 If $S^{0}$ is (weakly) multiplicative then $S^{0}$ is (weakly) perfect.

Proof. Suppose that $S^{0}$ is multiplicative and let $l \in \Lambda, i \in I$. Then $l i \in E^{0}$ and so $e_{l i}=f_{l i}=\overline{l i}$. A similar result holds for weakly multiplicative.

Proposition 5.2 If $S^{0}$ is perfect then $S^{0}$ is a quasi-ideal of $S$.

Proof. Since $e_{l i}=f_{l i} \in I \cap \Lambda=E^{0}$ then $l i \in R \cap L=S^{0}$ and $S^{0}$ is a quasi-ideal of $S$.

We can now deduce

Theorem 5.3 The following are equivalent: 
1. $S^{0}$ is multiplicative;

2. $S^{0}$ is weakly multiplicative and perfect;

3. $S^{0}$ is weakly multiplicative and a quasi-ideal of $S$.

Proof. $\quad(1) \Rightarrow(2)$ It is clear that multiplicative $\Rightarrow$ weakly multiplicative and the rest follows from Proposition 5.1.

$(2) \Rightarrow(3)$ This follows from Proposition 5.2.

(3) $\Rightarrow(1)$ Let $l \in \Lambda, i \in I$. Then $\overline{l i} \in E^{0}$ and $l i \in S^{0}$. Hence $\overline{l i}=l i$ and the result follows.

Theorem 5.4 $S^{0}$ is perfect if and only if $S^{0}$ is weakly perfect and a quasi-ideal of $S$.

Proof. If $S^{0}$ is perfect then it is a quasi-ideal of $S$. If $l \in \Lambda, i \in I$ then $e_{\overline{l i}}=\overline{e_{l i}}=\overline{f_{l i}}=f_{\overline{l i}}$ and so $S^{0}$ is weakly perfect.

Conversely, suppose that $S^{0}$ is weakly perfect and a quasi-ideal of $S$ and let $l \in \Lambda, i \in I$. Then $l i \in S^{0}$ and so $\overline{l i}=l i, e_{l i}=(l i)^{+}, f_{l i}=(l i)^{*}$ and $e_{\overline{l i}}=f_{\overline{l i}}$. But $(l i)^{+}=e_{\overline{l i}}$ and $(l i)^{*}=f_{\overline{l i}}$ and so $e_{l i}=f_{l i}$ and $S^{0}$ is perfect.

For inverse transversals, $S^{0}$ is a left simplistic transversal if and only if $\Lambda I \subseteq R$. We do not know whether this is true for adequate transversals but we can deduce

Proposition 5.5 The following are equivalent:

1. $S^{0}$ is weakly multiplicative and $\Lambda I \subseteq R$;

2. $\Lambda I \subseteq \Lambda$.

Proof. $\quad(1) \Rightarrow(2)$ If $l \in \Lambda, i \in I$ then $l i \in R$ and $\overline{l i} \in E^{0}$. Hence $l i=e_{l i} \overline{l i} f_{l i} \in E^{0} \Lambda \subseteq \Lambda$ as required.

(2) $\Rightarrow(1) \Lambda I \subseteq \Lambda \Rightarrow \overline{\Lambda I} \subseteq \bar{\Lambda}=E^{0}$ and so $S^{0}$ is weakly multiplicative. It is clear that $\Lambda I \subseteq R$.

Some of the properties considered so far can be summmarised by the following diagram, where for example, $l A=$ left adequate, $m=$ multiplicative etc.

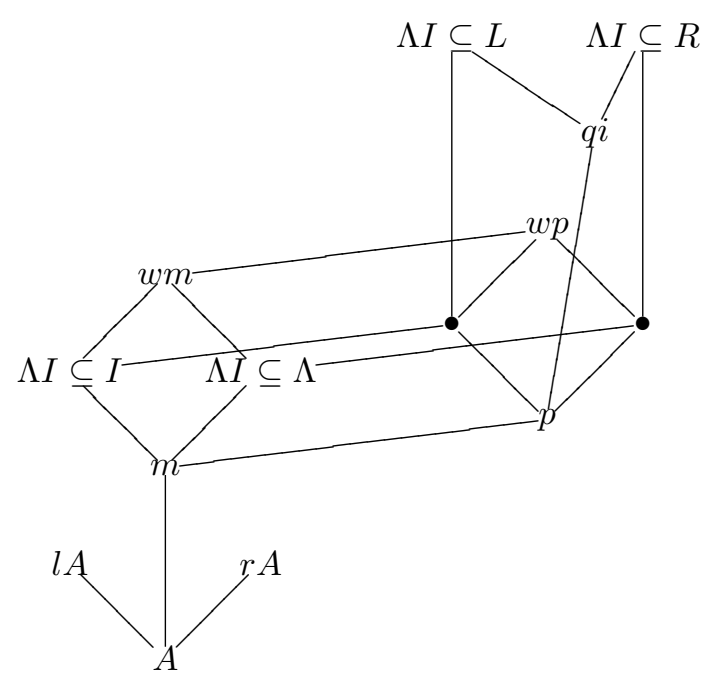




\section{Quasi-adequate semigroups}

In [3] the authors initiate the study of quasi-adequate semigroups and provide a structure theorem based on spined products. A semigroup is said to be quasi-adequate if it is abundant and its idempotents form a subsemigroup.

Proposition 6.1 [3, Proposition 1.3] Let $S$ be a semigroup and let $T$ be the set of regular elements of $S$. Then the following are equivalent:

1. $S$ is quasi-adequate;

2. $S$ is abundant and $T$ is an orthodox subsemigroup;

3. $T$ is an orthodox subsemigroup which has a non-empty intersection with each $\mathcal{L}^{*}$-class and each $\mathcal{R}^{*}$-class of $S$.

From section 2 we see that $U=T \cap S^{0}$ is an inverse transversal of $T$. The following now follows immediately from [1, Theorem 1.12].

Proposition 6.2 Let $S^{0}$ be an adequate transversal of an abundant semigroup $S$. Then the following are equivalent:

1. $S$ is quasi-adequate;

2. $(\forall x, y \in T),(x y)^{0}=y^{0} x^{0}$;

3. $(\forall i \in I)(\forall l \in \Lambda),(l i)^{0}=i^{0} l^{0}$;

4. $I \Lambda=E(S)$.

If $S$ is quasi-adequate then $T$ is orthodox and so $U$ is a weakly multiplicative inverse transversal of $T$ ([1, Theorem 2.9]). Hence:

Corollary 6.3 If $S$ is quasi-adequate then $S^{0}$ is weakly multiplicative.

Say that $S^{0}$ is left (right) perfect if for all $l \in \Lambda, i \in I, l i=\bar{i} l i \quad(l i=l i \bar{l})$ and that $S^{0}$ is weakly left (right) perfect if for all $l \in \Lambda, i \in I, \overline{l i}=\overline{(\bar{i} l i)} \quad(\overline{l i}=\overline{(l i \bar{l})})$.

Theorem 6.4 Let $S$ be a quasi-adequate semigroup. Then $S^{0}$ is perfect if and only if $S^{0}$ is both left and right perfect.

Proof. If $S^{0}$ is perfect then for all $l \in \Lambda, i \in I, f_{l i}=e_{l i}$. Hence from Theorem 3.11 $\bar{i} l i=l i \bar{l}$ and so $l i=(l i \bar{l})(\bar{l} \bar{i})(\bar{i} l i)=(l i \bar{l})(\bar{i} l i)=(l i \bar{l})(l i \bar{l})=l i l i \bar{l}=l i \bar{l}=\bar{i} l i$ and so $S^{0}$ is both right and left perfect. The converse is clear.

Notice that since $\overline{l i} \in E^{0}$ for all $l \in \Lambda, i \in I$, then $e_{\overline{l i}}=f_{\overline{l i}}=\overline{l i}$ and so we can deduce

Proposition 6.5 If $S$ is quasi-adequate then $S^{0}$ is weakly (left,right) perfect.

Theorem 6.6 Let $S$ be a quasi-adequate semigroup. The following are equivalent:

1. $S^{0}$ is left perfect;

2. $\Lambda I \subseteq \Lambda$;

3. $\Lambda I \subseteq R$; 


$$
\text { 4. } l \in \Lambda, i \in I,\left(e_{l i}, f_{l i}\right) \in \mathcal{R}^{*} \text {. }
$$

Proof. $\quad(1) \Rightarrow(2)$. If $S^{0}$ is left perfect then $l i=f_{l i}$ and so $l i \in \Lambda$.

$(2) \Rightarrow(3)$. Obvious.

(3) $\Rightarrow(4)$. If $\Lambda I \subseteq R$ then $\Lambda I \subseteq \Lambda=E \cap R$. Let $l \in \Lambda, i \in I$. Then $e_{l i} f_{l i}=l i \bar{i} l i=l i=f_{l i}$. Also $f_{l i} e_{l i}=\bar{i} l i l i \bar{l}=\bar{i} l i \bar{l}=f_{l i} \bar{l}=l i \bar{l}=e_{l i}$ and $\left(e_{l i}, f_{l i}\right) \in \mathcal{R}^{*}$ as required.

$(4) \Rightarrow(1)$. If $\left(e_{l i}, f_{l i}\right) \in \mathcal{R}^{*}$ then $e_{l i} f_{l i}=f_{l i}$ and so $l i=l i l i=l i \bar{l} l i=f_{l i} l i=e_{l i} f_{l i} l i=e_{l i} l i=$ $\bar{i} l i l i=\bar{i} l i$ and $S^{0}$ is left perfect.

Notice that if $S$ is quasi-adequate then $\Lambda I \subseteq E$. If in addition $S$ is left adequate then $E=I$ and so $S^{0}$ is right perfect. Hence we have:

Theorem 6.7 If $S$ is quasi-adequate and left adequate then $S^{0}$ is right perfect.

Proposition 6.8 If $S$ is quasi-adequate then $S^{0}$ is weakly left (right) perfect if and only if $S^{0}$ is weakly perfect.

Proof. If $S^{0}$ is weakly left perfect then for all $l \in \Lambda, i \in I$ we have $\overline{l i}=\overline{\bar{i} l i}=\overline{f_{l i}} \in E^{0}$ and so $S^{0}$ is weakly multiplicative and hence weakly perfect.

Theorem 6.9 Let $S$ be quasi-adequate. Then $S^{0}$ is left simplistic if and only if $E^{0}$ is a right ideal of $I$.

Proof. One way round follows from Proposition 4.5. Suppose then that $S^{0}$ is left simplistic. Then $I$ is a band from Theorem 2.5. Also $E^{0} I \subseteq S^{0}$ but as $E^{0} I \subseteq E$ then the result follows.

Theorem 6.10 Suppose that $S$ is quasi-adequate. If $S^{0}$ is left perfect then $S^{0}$ is left simplistic.

Proof. Let $e \in E^{0}$ and $i \in I$. Then $e i=\bar{i} e i=e \bar{i} i=e \bar{i}$ and so from Theorem 4.2 it follows that $E^{0}$ is a right ideal of $I$.

From these results the previous diagram now reduces to

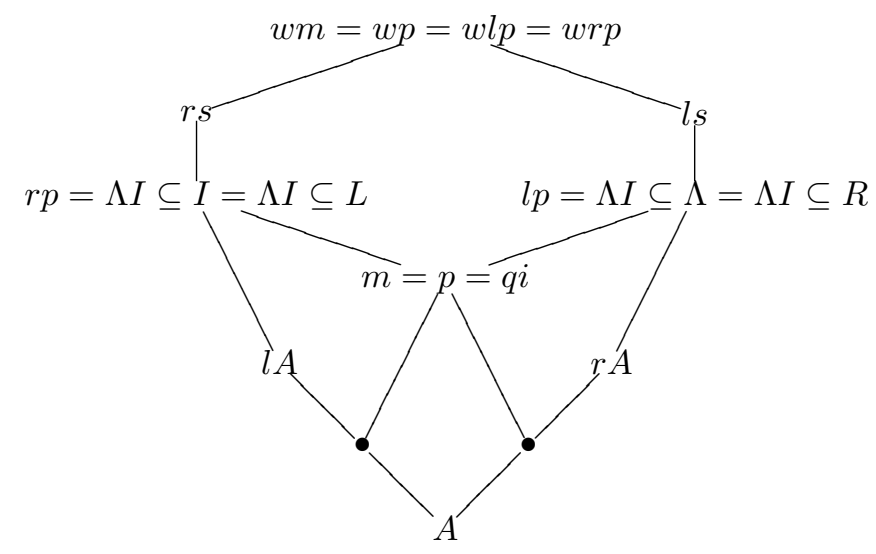




\section{Monoids}

If $S$ is a monoid with identity 1 , notice that $1=e_{1} \overline{1} f_{1}$ and that $e_{1} \mathcal{R}^{*} 1$. Hence $e_{1} \mathcal{R} 1$ and so $e_{1} .1=1$. Similarly, $1=f_{1}$ and so $1=\overline{1}$ and so $1 \in S^{0}$.

Theorem 7.1 If $S$ is a monoid then $S$ is right adequate if and only if $S^{0}$ is left simplistic.

Proof. Suppose that $S^{0}$ is left simplistic then for all $i \in I$ it follows that $i=1 . i \in E^{0} I \subseteq$ $S^{0}$. Hence $I=E^{0}$ and $S$ is right adequate from the dual of Theorem 3.14. Conversely, if $S$ is right adequate then $I=E^{0}$ and so $E^{0} I=E^{0} \subseteq S^{0}$ and so $S^{0}$ is left simplistic.

Notice that the conclusions of the previous theorem hold for any semigroup in which $I \subseteq E^{0} I$.

It is easy to demonstrate that similar conclusions hold if $S$ is such that $\Lambda I \subseteq R$ and if $S$ is such that $E^{0}$ is a right ideal of $I$ and if $S^{0}$ is left perfect.

Theorem 7.2 If $S$ is a monoid then $S$ is adequate if and only if $S^{0}$ is multiplicative if and only if $S^{0}$ is a quasi-ideal if and only if $S^{0}$ is perfect.

Proof. We know that if $S^{0}$ is multiplicative then $S^{0}$ is a quasi-ideal and that $S^{0}$ is a quasi-ideal if and only if $S$ is adequate (from Theorem 7.1 and its dual).

If $S^{0}$ is a quasi-ideal then from Theorem 7.1 and Theorem 3.14 it follows that $I=\Lambda=E^{0}$ and so $\Lambda I \subseteq E^{0}$ and so $S^{0}$ is multiplicative.

If $S$ is adequate then $S^{0}$ is multiplicative and so $S^{0}$ is perfect. Conversely, if $S^{0}$ is perfect then $S^{0}$ is a quasi-ideal and so $S$ is adequate.

Consequently we have a diagram

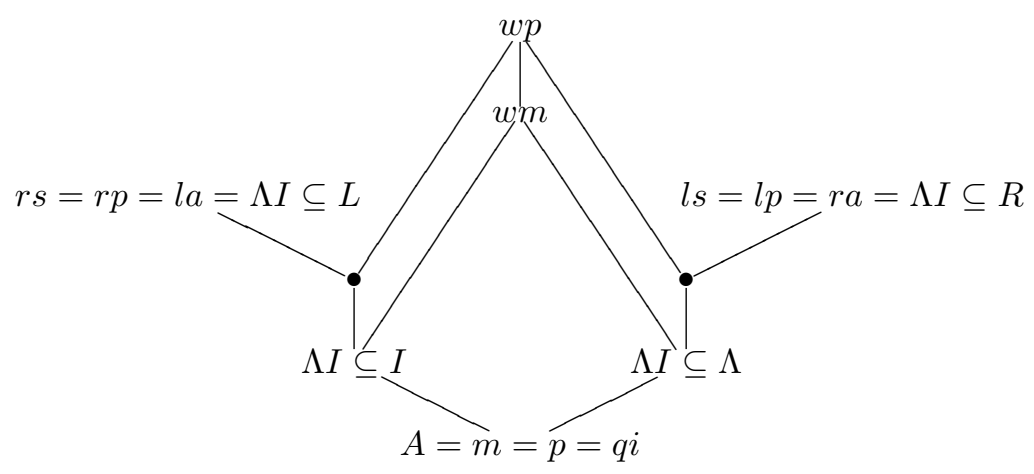

\section{References}

[1] T.S. Blyth, 'Inverse Transversals - A Guided Tour' in Proceedings of the International Conference on Semigroups, Braga Portugal 18-23 June 1999, Ed. Paula Smith, Emilia Giraldes, Paula Martins, World Scientific (2000) 26-43.

[2] Jianfei Chen, Abundant Semigroups with Adequate Transversals, Semigroup Forum, Vol. 60 (2000) 67-79.

[3] A. El-Qallali and J.B. Fountain, Quasi-adequate semigroups, Proceedings of the Royal Society of Edinburgh, 91A, 91-99, 1981. 
[4] Abdulsalam El-Qallali, Abundant Semigroups with a multiplicative Type A transversal, Semigroup Forum, Vol. 47 (1993) 327-340.

[5] John Fountain, Adequate Semigroups, Proceedings of the Edinburgh Mathematical Society (1979), 22 113-125.

[6] John Fountain, Abundant Semigroups, Proc. London Math. Soc. 44 (1982), 103-129.

[7] Xiaojiang Guo and Li-Ming Wang, Idempotent-connected abundant semigroups which are disjoint unions of quasi-ideal adequate transversals, Communications in Algebra, Vol. 30 No. 4 (2002) 1779 - 1800.

[8] Howie, J.M., Fundamentals of Semigroup Theory, London Mathematical Society Monographs, (OUP, 1995).

[9] Minxia Luo, Relationships between the Quasi-ideal Adequate Transversals of an Abundant Semigroup, Semigroup Forum, Vol. 67 (2003) 411-418.

[10] Zhang Ronghua and Wang Shoufeng, On Generalized Adequate Transversals, Communications in Algebra, Vol. 34 No. 7 (2006), 2419 - 2436. 